\section{Prevalência de diabetes mellitus e fatores associados em mulheres indígenas do Município de Dourados, Mato Grosso do Sul, Brasil}

\author{
Prevalence of diabetes mellitus and associated \\ factors in indigenous women in Dourados, \\ Mato Grosso do Sul State, Brazil \\ Prevalencia de diabetes mellitus y factores \\ asociados en mujeres indígenas del municipio de \\ Dourados, Mato Grosso do Sul, Brasil
}

\begin{abstract}
Resumo
O objetivo do estudo foi estimar a prevalência de glicemia capilar casual alterada, sugestiva de diabetes mellitus, e sua associação com os fatores socioeconômicos, demográficos, antropométricos e clínicos em mulheres indígenas do Município de Dourados, Mato Grosso do Sul, Brasil. Trata-se de um estudo transversal, com amostra probabilística composta por mulheres de 18-59 anos, residentes nas aldeias Bororó e Jaguapiru. Foi utilizado um questionário padronizado para a coleta das informações, $e$ a análise estatística, realizada por meio dos testes Kruskal Wallis, Mann Whitney e do modelo linear generalizado. Entre as 385 mulheres, 7\% apresentaram glicemia capilar casual alterada sugestiva de diabetes mellitus. No modelo linear generalizado, permaneceram associadas, no modelo final, as variáveis idade $(p<0,001)$, pressão arterial sistólica $(p=0,002) e$ etnia $(p=0,022)$. Este estudo demonstra a necessidade de mudanças comportamentais, com o auxílio de intervenções educativas como estratégia de prevenção e controle do diabetes mellitus.
\end{abstract}

Hiperglicemia; Diabetes Mellitus; Saúde da Mulher; População Indígena
Glênio Alves de Freitas 1

Maria Cristina Corrêa de Souza 1

Rosangela da Costa Lima 1 


\section{Introdução}

O diabetes mellitus é considerado um dos grandes problemas de saúde pública do século XXI. Fatores como envelhecimento populacional, sedentarismo, hábitos alimentares inadequados e avanços terapêuticos para o tratamento da doença estão contribuindo para o aumento do número de casos 1 .

No Brasil, a prevalência de diabetes mellitus é de 9,19\%, sendo o $6^{\circ}$ país no ranking mundial de óbitos em consequência da doença ${ }^{2}$. A pesquisa Vigilância de Fatores de Risco e Proteção para Doenças Crônicas por Inquérito Telefônico (VIGITEL), realizada nas capitais brasileiras, revelou um aumento de aproximadamente $40 \%$ na prevalência de diabetes mellitus entre 2006 e 20123.

Na população indígena, o diabetes mellitus é uma doença emergente. Além dos fatores de risco tradicionais, problemas sociais associados às alterações da economia de subsistência, ao consumo de alimentos industrializados e ao contato cada vez mais frequente com a população urbana contribuem para o aparecimento dessa e de outras doenças crônicas não transmissíveis (DCNT) 4 .

Em vários países, a prevalência de diabetes mellitus entre os indígenas merece destaque. No Canadá, em um estudo realizado com os indígenas Metis com idade superior a 19 anos, a prevalência de diabetes mellitus foi de $11,8 \% 5$. No México, os indígenas Pima de Sonora apresentaram uma prevalência de $6,3 \%$ e $10,5 \%$, entre homens e mulheres, respectivamente, enquanto que, nos Estados Unidos, 50\% dos indígenas, acima dos 35 anos, dessa mesma etnia, eram diabéticos 6,7. Indígenas que possuem o estilo de vida tradicional ainda apresentam baixas prevalências de diabetes mellitus, como os Ayamara do Chile, onde apenas $1 \%$ da população possui diabetes mellitus ${ }^{8}$.

Entre os indígenas no Brasil, o diabetes mellitu era desconhecido até a década de 19704 . O primeiro estudo que investigou a ocorrência de diabetes mellitus ocorreu em 1975 com indígenas do Norte do Pará, não sendo encontrado nenhum caso 9. Em 1977, os indígenas do Norte do Amapá foram avaliados, e $1 \%$ dos indivíduos apresentou diabetes mellitus 10 . Na aldeia Jaguapiru, localizada no Município de Dourados, Mato Grosso do Sul, Região Centro-oeste do Brasil, um estudo realizado entre 2007 e 2008 detectou que $6,8 \%$ das mulheres possuíam diabetes mellitus 11 . Outra avaliação realizada na mesma aldeia, entre 2009 e 2011, observou que a prevalência de diabetes mellitus, no mesmo sexo, elevouse para $7,8 \% 12$. Mais recentemente, os indígenas Xavante do Estado do Mato Grosso, Região Cen-
tro-Oeste do Brasil, apresentaram prevalência de diabetes mellitus de $28,2 \%$ na população geral e $40,3 \%$ nas mulheres 13 . No I Inquérito Nacional de Saúde e Nutrição Indígena, realizado entre 2008 e 2009, 1,4\% das mulheres indígenas brasileiras apresentou valores de glicemia sugestivos de diabetes mellitus. A Região Norte, que possui indígenas com contato recente com a população urbana, apresentou a menor prevalência $(0,5 \%)$, enquanto que as regiões Sul/Sudeste apresentaram o maior percentual $(2,1 \%) 14$.

A reserva de Dourados, possui cerca de $12 \mathrm{mil}$ indígenas subsistindo em situação de pobreza, marginalidade, desintegração social e violência 15,16 . Para garantir a renda familiar para a sobrevivência, a maioria dos homens trabalha nas usinas de açúcar e álcool da região em condições insalubres 16. Entre as mulheres, grande parte permanece na reserva cuidando dos filhos, e a minoria trabalha nas escolas ou como agentes de saúde nas aldeias 16. A alta densidade populacional e as dificuldades vivenciadas por esses indígenas têm contribuído para afetar seu modo de vida tradicional 17 .

Dado ao aumento da prevalência de diabetes mellitus na população indígena ao longo dos anos, além da existência da escassez de estudos locais que permitam a comparação com os dados regionais e nacionais, o objetivo deste trabalho foi estimar a prevalência de diabetes mellitus, por meio da glicemia capilar casual, e os fatores associados em mulheres indígenas do Município de Dourados.

\section{Material e métodos}

\section{Delineamento e população}

Foi realizado um estudo transversal, com amostra probabilística composta por mulheres indígenas com idade entre 18 e 59 anos, residentes nas aldeias participantes. A reserva indígena do Município de Dourados localiza-se nos limites da cidade de Dourados. É composta pelas aldeias Jaguapiru e Bororó, sendo a primeira habitada pelos indígenas Terena e Guarani (Kaiowá e Nandeva), e a segunda habitada majoritariamente pelos indígenas Guarani 18.

O cálculo do tamanho de amostra foi realizado no programa Epi Info, versão 3.5.4 (Centers for Disease Control and Prevention, Atlanta, Estados Unidos), com os seguintes parâmetros: prevalência de $4 \%$ - média dos resultados dos estudos regionais da aldeia Jaguapiru $(6,8 \%)$ e Região Centro-Oeste $(1,4 \%)$ 11,14, considerando 1.714 mulheres com idade entre 18 e 59 anos na aldeia Jaguapiru e 1.538 na Bororó 19; nível de 
95\% de confiança e o erro de três pontos percentuais. A amostra para a aldeia Jaguapiru e Bororó foi de 150 e 149 mulheres, respectivamente. Com a inclusão de $20 \%$ de perdas e recusas, a amostra foi ampliada para 359.

O sorteio dos domicílios ocorreu de forma aleatória simples, pelo programa IBM SPSS, versão 21 (IBMCorp., Armonk, Estados Unidos), com os domicílios georreferenciados pelo Curso de Geografia da Universidade Federal da Grande Dourados (UFGD). Para a localização dos domicílios, foi utilizado o mapa das aldeias e o uso do sistema de posicionamento global (GPS) da marca GarmineTrex (Garmin Ltda, Kansa City, Estados Unidos). Foram encontrados 435 (87\%) dos domicílios sorteados, e todas as mulheres residentes foram convidadas a participar do estudo. Não foram encontrados geograficamente 65 domicílios. Mulheres não indígenas que residiam nas aldeias e gestantes foram excluídas.

\section{Instrumentos e coleta de dados}

Foram utilizados dois questionários pré-codificados e padronizados, elaborados a partir do $I$ Inquérito Nacional de Saúde Indígena ${ }^{14}$. As variáveis coletadas incluíram: (1) demográficas e nível socioeconômico: idade (data de nascimento), etnia referida, escolaridade (em anos completos). Para a classificação do nível econômico, foi realizada uma análise fatorial com base na matriz de correlações de 19 bens duráveis 14 (rádio, geladeira ou freezer, videocassete ou DVD, fogão a gás, máquina de lavar roupa, forno de micro-ondas, linha de telefone fixo, telefone celular, computador, motosserra, motocicleta/ mobilete, animais de carga ou trabalho, motores de popa, televisões, automóveis, aparelhos de ar-condicionado, antenas parabólicas, bicicletas e raladores de mandioca). O item ralador de mandioca foi excluído da análise devido a sua ausência em todos os domicílios estudados. Assim, foi realizada uma matriz de correlações de 18 bens duráveis. As correlações variaram entre $-0,95$ (animal de carga x computador) e 0,48 (televisão x videocassete/DVD). O teste Kaiser-Meyer-Olkin (KMO) atingiu valor de 0,75, indicando adequação da amostra para a análise proposta. A extração resultou em 8 componentes. $\mathrm{O}$ valor inicial do primeiro componente foi 3,22, sendo responsável por 17,91\% da variância. Já o segundo componente obteve valor inicial de 1,59 com variância correspondente a 8,81\%. A partir do segundo componente, verificou-se discreta diferença entre as variâncias. Apenas o primeiro componente foi considerado na classificação socioeconômica, com exclusão das variáveis com correlação $<1$ (animal de carga, motor de popa, telefone fixo, ar-condicionado). O resultado da análise de componentes principais gerou um valor para cada bem durável que foi multiplicado pelo número de itens em cada domicílio. Posteriormente, esse escore foi analisado em 3 categorias de acordo com a medida separatriz tercil; (2) hábitos de vida: tabagismo (“A senhora fuma?") e consumo de bebida alcoólica ("A senhora faz uso de bebidas alcoólicas?”); (3) clínicas: uso de medicações para diabetes mellitus, aferição da pressão arterial, medidas antropométricas e dosagem de glicemia capilar casual.

A pressão arterial foi aferida em dois momentos, com intervalo mínimo de 5 minutos, sendo utilizada, para a análise, a média aritmética dessas. A aferição foi realizada no pulso, pelo tensiômetro automático Omrom, modelo Hem-631INT (Omron Healthcare, São Paulo, Brasil), devidamente calibrado. Caso a entrevistada apresentasse bexiga cheia ou tivesse realizado esforço físico, consumido café e/ou uso de tabaco até 30 minutos antes da primeira aferição, ela era aconselhada a esvaziar a bexiga, sendo aguardados 30 minutos para realizar a primeira aferição. A entrevistada foi orientada a retirar os adereços e a não falar durante o procedimento. Durante o processo, a entrevistada ficou sentada, com os pés apoiados no chão e o braço sobre a mesa para a fixação do manguito no pulso. Posteriormente, o braço foi flexionado com o pulso à altura do coração com a palma da mão voltada para o peito. Foram consideradas hipertensas as mulheres que possuíam pressão sistólica $\geq 140 \mathrm{mmHg}$ e/ou pressão diastólica $\geq 90 \mathrm{mmHg} 20$.

O peso das entrevistadas foi coletado com as participantes descalças e utilizando roupas leves. Foi utilizada a balança Marte do modelo LC200PP (Marte Científica, São Paulo, Brasil), cuja capacidade máxima é de $200 \mathrm{~kg}$ e precisão de 50g. A estatura foi mensurada a partir do estadiômetro portátil Alturaexata (AlturaExata, Minas Gerais, Brasil), com capacidade máxima de $213 \mathrm{~cm}$ e resolução de $1 \mathrm{~mm}$, com a mulher na posição ereta, descalça e sem adereços no cabelo, com a região posterior em contato com a régua do equipamento.

O índice de massa corporal (IMC) foi calculado como peso em quilogramas $(\mathrm{kg})$, dividido pela altura em metros ao quadrado $\left(\mathrm{m}^{2}\right)$. O IMC foi avaliado conforme a classificação da Organização Mundial da Saúde (OMS) 21.

A circunferência da cintura (CC) foi medida em centímetros $(\mathrm{cm})$, utilizando uma trena antropométrica inextensível da marca Cescorf (Cescorf Equipamentos Antropométricos, Porto Alegre, Brasil), com 2m de comprimento, no ponto médio entre a última costela e a parte superior da crista ilíaca, com as entrevistadas em pé. $\mathrm{O}$ 
ponto de corte para risco de doenças cardiovasculares foi de $80-87,9 \mathrm{~cm}$ (risco elevado) e $\geq 88 \mathrm{~cm}$ (risco muito elevado) para mulheres 21 .

Para a dosagem da glicemia capilar casual, foi utilizado aparelho Test Line (TestLine Clinical Diagnostics, Brno, Czech Republic) com lancetas e fitas reagentes. Apesar de não ser um teste padrão ouro, esse método foi selecionado pela facilidade de realização no trabalho de campo.

A entrevistada foi orientada, antes da realização do procedimento, a lavar as mãos com água e sabão. Após a lavagem, o entrevistador escolheu uma polpa digital livre de calosidades ou de lesões contaminadas. Foi realizada a antissepsia com álcool 70\% no local, e, posteriormente, feita a punção. Após a punção, a gota de sangue foi retirada para análise. O valor de referência utilizado para glicemia casual alterada foi $\geq$ $200 \mathrm{mg} / \mathrm{dL} 22$

Considerando a possibilidade de algumas mulheres indígenas já possuírem o diagnóstico prévio de diabetes mellitus com uso de hipoglicemiantes (insulina e/ou hipoglicemiante oral), foi perguntado durante a entrevista se as mulheres utilizavam alguma medicação para diabetes mellitus.

Para a prevalência de glicemia casual alterada sugestiva de diabetes mellitus, foram consideradas as mulheres com glicemia capilar casual $\geq$ $200 \mathrm{mg} / \mathrm{dL}$ e/ou aquelas que referiram o uso de medicamentos para diabetes mellitus, mesmo com o valor da glicemia $\leq 200 \mathrm{mg} / \mathrm{dL}$ (glicemia capilar casual alterada corrigida pelo uso de hipoglicemiante ou insulina).

As mulheres que apresentaram alteração na pressão arterial ou na glicemia capilar casual foram encaminhadas para o posto de saúde de referência.

A equipe de coleta de dados foi composta por dois entrevistadores e um tradutor nativo da aldeia. Os entrevistadores eram alunos do curso de Mestrado em Ciências da Saúde e do curso de Nutrição da UFGD. Foi elaborado um manual de técnicas, e a equipe realizou oficinas para o treinamento e a padronização para a entrevista e a coleta dos dados clínicos. Durante as visitas, caso o domicílio estivesse fechado, o entrevistador retornava até três vezes ao domicílio em horários diferentes.

\section{Análise estatística}

Os entrevistadores realizaram a codificação dos questionários que, posteriormente, foram revisados e tabulados pelo supervisor. O banco de dados foi duplamente digitado no programa Epi Data, versão 3.1 (Epidata Assoc., Odense, Dinamarca). A análise de dados foi realizada no programa IBM SPSS, versão 21. A variável dependente glicemia capilar casual foi testada quanto a sua normalidade pelo teste de Kolmogorov-Smirnov. As variáveis numéricas que apresentaram distribuição normal foram descritas por meio da média (desvio padrão) e as que não apresentaram distribuição normal pela mediana (intervalo interquartil). As variáveis categóricas foram descritas pela proporção. A comparação entre as medianas foi realizada pelos testes de Mann-Whitney e Kruskal Wallis. O modelo linear generalizado, com distribuição Gama e função de ligação identidade, foi utilizado para verificar a associação entre glicemia capilar casual (variável contínua) e demais características. Iniciou-se a formação dos modelos com um modelo maximal com a exclusão das variáveis com o maior valor de $\mathrm{p}$, permanecendo somente aquelas que realmente se ajustaram ao modelo (seleção backward) 23.

\section{Considerações éticas}

O estudo foi aprovado pelo Comitê de Ética em Pesquisa da UFGD (CEP/UFGD - Protocolo 009/2011) e pela Comissão Nacional de Ética em Pesquisa (CONEP - parecer no 653/2011). As lideranças indígenas e o Conselho Distrital de Saúde Indígena assinaram a carta de anuência para a realização da pesquisa. Todas as participantes assinaram o Termo de Consentimento Livre e Esclarecido.

\section{Resultados}

A coleta de dados foi realizada no período de junho a outubro de 2013. Se recusaram a participar do estudo 24 mulheres, e, em 26 domicílios, as moradoras não foram encontradas após 3 visitas. $\mathrm{O}$ estudo foi realizado com 385 indígenas, a amostra foi superior à calculada, uma vez que este estudo faz parte de um projeto intitulado "Perfil de saúde e nutrição de indígenas de Dourados, Mato Grosso do Sul”. A aldeia Jaguapiru apresentou o maior percentual de participantes $(51,6 \%)$, a etnia Guarani foi predominante $(77,4 \%)$, a média de idade geral foi de $34,5( \pm 11,0)$, e metade das entrevistadas possuía até quatro anos de estudo. Quando avaliados os hábitos de vida, aproximadamente, $10 \%$ referiram tabagismo e consumo de bebida alcoólica. A média do IMC foi de $27,85( \pm 5,02) \mathrm{kg} / \mathrm{m}^{2}$, e da circunferência da cintura, 90,29 $( \pm 11,61) \mathrm{cm}$ (Tabela 1$)$.

Foi detectado sobrepeso em $40,3 \%$ das indígenas e obesidade em 30,9\%. Adiposidade abdominal, indicando risco muito alto para doenças cardiovasculares, esteve presente em $57,7 \%$ das 
Características sociodemográficas, econômicas, antropométricas e clínicas de mulheres da reserva indígena de Dourados, Mato Grosso do Sul, Brasil $(n=385)$

\begin{tabular}{|c|c|}
\hline Variáveis & Valores \\
\hline \multicolumn{2}{|l|}{ Aldeia (\%) } \\
\hline Bororó & 48,6 \\
\hline Jaguapiru & 51,4 \\
\hline \multicolumn{2}{|l|}{ Etnia (\%) } \\
\hline Guarani & 77,4 \\
\hline Terena & 22,6 \\
\hline Idade média (DP) & $34,5(11,0)$ \\
\hline \multicolumn{2}{|l|}{ Escolaridade [anos] (\%) } \\
\hline Nenhum & 14,0 \\
\hline $1-4$ & 41,8 \\
\hline $5-9$ & 22,9 \\
\hline$>10$ & 21,3 \\
\hline \multicolumn{2}{|l|}{ Nível socioeconômico (\%) } \\
\hline Tercil 1 & 33,0 \\
\hline Tercil 2 & 32,2 \\
\hline Tercil 3 & 34,8 \\
\hline Tabagismo (\%) & 11,9 \\
\hline Consumo de bebida alcoólica (\%) & 10,9 \\
\hline IMC [kg/m²] média (DP) & $27,8(5,0)$ \\
\hline CC [cm] média (DP) & $90,2(11,6)$ \\
\hline \multicolumn{2}{|l|}{ Pressão arterial [mmHg] média (DP) } \\
\hline Sistólica & $131,4(23,7)$ \\
\hline Diastólica & $83,8(15,8)$ \\
\hline Histórico familiar de diabetes mellitus (\%) & 20,0 \\
\hline Glicemia casual capilar, mg/dL [mediana] (P25-P75) & $106,0(95,5-126,0)$ \\
\hline Glicemia capilar casual alterada sugestiva de diabetes mellitus (\%) * & 7,0 \\
\hline
\end{tabular}

CC: circunferência da cintura; DM: diabetes mellitus; DP: desvio padrão; IMC: índice de massa corporal.

* Glicemia capilar casual > 200mg/dL e/ou glicemia capilar casual alterada corrigida pelo uso de hipoglicemiantes ou insulina.

mulheres estudadas, e hipertensão arterial sistêmica, em 37,7\% (dados não tabulados).

A presença de familiares de primeiro grau com diabetes mellitus foi referida por $20 \%$ das mulheres do estudo, e o uso de medicamentos para o diabetes mellitus, por 5,5\% (dados não tabulados). A mediana de glicemia capilar casual foi de 106,0mg/dL. A glicemia capilar casual alterada, mesmo com o uso de medicamento para diabetes mellitus, esteve presente em 3,6\% das participantes (dados não tabulados). A prevalência de glicemia casual alterada sugestiva de diabetes mellitus (mulheres com glicemia casual alterada e/ou glicemia casual alterada corrigida pelo uso de hipoglicemiantes ou insulina) foi de 7\% (Tabela 1). A aldeia Bororó apresentou uma prevalência de 2,9\%, e a aldeia Jaguapiru, de 4,1\% (dados não tabulados). A glicemia capilar causal $<200 \mathrm{mg} / \mathrm{dL}$ foi encontrada em $62 \%$ das mulhe- res que utilizavam medicamentos para diabetes mellitus. Dentre as indígenas que apresentaram glicemia capilar casual alterada, $42,8 \%$ não faziam uso de medicamentos para o diabetes mellitus, e 57,1\% possuíam entre 40 e 49 anos (dados não tabulados).

A Tabela 2 demonstra as medianas de glicemia capilar casual de acordo com as características sociodemográficas, econômicas, hábitos de vida, antropométricas e clínicas. Não houve diferenças estatisticamente significativas nas medianas de glicemia conforme aldeia e etnia. As medianas de glicemia capilar casual aumentaram proporcionalmente com a idade ( $p$ $<0,001)$ e IMC ( $\mathrm{p}=0,035)$ e são inversamente proporcionais à escolaridade $(\mathrm{p}=0,001)$. Indígenas que apresentaram pressão arterial sistólica (PAS - p $<0,001)$ e pressão arterial diastólica $(\mathrm{PAD}-\mathrm{p}=0,007)$ elevadas e referiram o uso de 
Tabela 2

Medianas (P25-P75) de glicemia capilar casual conforme características sociodemográficas, econômicas, hábitos de vida, antropométricas e clínicas de mulheres da reserva indígena de Dourados, Mato Grosso do Sul, Brasil $(n=385)$.

\begin{tabular}{|c|c|}
\hline Variáveis & $\begin{array}{c}\text { Valor de } \mathrm{p} \\
\text { Mediana (P25-P75) }\end{array}$ \\
\hline Aldeia & $p=0,659$ * \\
\hline Bororó & $107,0(96,0-127,0)$ \\
\hline Jaguapiru & $106,0(94,0-106,0)$ \\
\hline Etnia & $p=0,662 * \star$ \\
\hline Guarani & $105,0(95,0-126,0)$ \\
\hline Terena & $110,0(96,0-123,0)$ \\
\hline Idade [anos] & $p<0,001 * *$ \\
\hline $18-29$ & $100,5(91,0-116,2)$ \\
\hline $30-39$ & $106,0(95,0-119,0)$ \\
\hline $40-49$ & $119,0(100,0-142,0)$ \\
\hline $50-59$ & $119,0(102,0-150,0)$ \\
\hline Escolaridade [anos] & $p=0,001 * \star$ \\
\hline Nenhum & $119,0(99,7-136,5)$ \\
\hline $1-4$ & $108,0(97,0-129,0)$ \\
\hline $5-9$ & $101,0(94,0-119,0)$ \\
\hline$>10$ & $101,0(93,7-119,2)$ \\
\hline Nível socioeconômico & $p=0,423 * \star$ \\
\hline Tercil 1 & $107,0(96,0-129,0)$ \\
\hline Tercil 2 & $105,5(92,5-122,0)$ \\
\hline Tercil 3 & $108,0(96,7-123,5)$ \\
\hline Tabagismo & $p=0,901$ * \\
\hline Sim & $109,0(92,7-125,0)$ \\
\hline Não & $106,0(96,0-126,0)$ \\
\hline Consumo de bebida alcoólica & $p=0,236$ * \\
\hline Sim & $110,0(96,7-138,0)$ \\
\hline Não & $106,0(95,0-124,0)$ \\
\hline $\mathrm{IMC}\left[\mathrm{kg} / \mathrm{m}^{2}\right]$ & $p=0,035$ * \\
\hline$<24,9$ & $105,0(92,7-123,2)$ \\
\hline $25,0-29,9$ & $103,0(95,0-122,0)$ \\
\hline$>30$ & $115,0(98,0-130,0)$ \\
\hline $\mathrm{CC}[\mathrm{cm}]$ & $p=0,106 * *$ \\
\hline$<80$ & $103,0(91,0-120,0)$ \\
\hline $80-87$ & $103,0(92,2-117,7)$ \\
\hline$>88$ & $108,5(96,7-129,0)$ \\
\hline PAS [mmHg] & $p<0,001$ * \\
\hline$<140$ & $103,0(94,0-119,5)$ \\
\hline$>140$ & $115,0(99,0-138,0)$ \\
\hline PAD $[\mathrm{mmHg}]$ & $p=0,007$ * \\
\hline$<90$ & $109,0(94,0-121,0)$ \\
\hline$>90$ & $114,0(98,0-136,0)$ \\
\hline Histórico familiar de diabetes mellitus & $p=0,735$ * \\
\hline Sim & $108,0(95,0-121,5)$ \\
\hline
\end{tabular}

CC: circunferência da cintura; DM: diabetes mellitus; IMC: índice de massa corporal; PAD: pressão arterial diastólica; PAS: pressão arterial sistólica.

*Teste de Mann Whitney;

** Teste de Kruskal Wallis. 
medicamentos para diabetes $(\mathrm{p}<0,001)$ apresentaram maiores medianas de glicemia (Tabela 2).

A Tabela 3 apresenta o modelo linear generalizado. A associação entre a glicemia capilar casual e a idade demonstrou que, a cada ano, ocorre o aumento de $0,74 \mathrm{mg} / \mathrm{dL}$ de glicemia $(\mathrm{p}<$ 0,001). Em relação às etnias, a Terena apresentou um aumento de $9,54 \mathrm{mg} / \mathrm{dL}$ de glicemia capilar casual quando comparada à Guarani $(p=0,022)$. A pressão arterial sistólica também demonstrou associação com a glicemia capilar casual. Para o aumento de $1 \mathrm{mmHg}$ de pressão arterial sistólica, ocorreu um aumento de $0,30 \mathrm{mg} / \mathrm{dL}$ de glicemia capilar casual $(\mathrm{p}=0,002)$.

\section{Discussão}

A prevalência de glicemia casual alterada sugestiva de diabetes mellitus na reserva indígena de Dourados, (7\%) aproximou-se das prevalências de diabetes mellitus dos estudos realizados anteriormente na aldeia Jaguapiru que apresentaram, entre as mulheres, taxas de 6,8\% e 7,8\% 11,12 . No entanto, no estudo atual, a aldeia Jaguapiru apresentou uma prevalência superior $(4,1 \%)$ quando comparada com a aldeia Bororó $(2,9 \%)$. Essa diferença pode estar relacionada a questões étnicas e ambientais. Na aldeia Bororó, 97\% dos indígenas são da etnia Guarani, enquanto que, na aldeia Jaguapiru, essa etnia representa 58\% dos habitantes 18. Os Guaranis, ao contrário dos Terenas, possuem uma forte ligação com o território em que vivem, o que contribui tanto para a preservação da cultura tradicional, quanto para a redução da ocorrência de doenças crônicas não transmissíveis devido ao pouco contato com a sociedade envolvente 17 . As prevalências dos valores de glicose sugestivos de diabetes mellitus em mulheres indígenas em nível nacional $(1,4 \%)$ e da Região Centro-oeste $(1,4 \%)$, determinadas no I Inquérito de Saúde e Nutrição Indígena, foram inferiores aos resultados aqui encontrados. É importante ressaltar que, no estudo, em nível nacional, foram avaliados indígenas de várias etnias, com diferentes características socioculturais e econômicas 14 .

No estudo realizado com os indígenas Yanomami, no Estado de Roraima, com amostragem de conveniência, apenas um caso $(1,4 \%)$ de glicemia alterada foi encontrado ${ }^{24}$. No entanto, a prevalência, no presente estudo, foi semelhante à encontrada nos indígenas Khisedje $(3,8 \%)$, do Parque Xingu, na Região Central do Brasil 25. Quando comparado com os indígenas norte-americanos Cocopah (34\%) e Cherokee (10\%), a prevalência de glicemia capilar casual alterada foi inferior. Além das características étnicas e ambientais, os norte-americanos tiveram um contato intenso e precoce com os povos não indígenas, o que contribuiu para a modificação dos hábitos tradicionais 26,27 .

Dentre as mulheres com glicemia capilar casual alterada, 42,8\% não faziam uso de medicações para diabetes mellitus. Fato semelhante foi observado nos Estados Unidos, entre os anos de 2003 e 2004, onde 39,4\% dos diabéticos desconheciam o diagnóstico 28 . A baixa escolaridade as dificuldades na comunicação e a falta de profissionais preparados nos serviços de saúde para atender à população indígena podem contribuir tanto para o subdiagnóstico quanto para o tratamento inadequado do diabetes mellitus.

A ocorrência do diabetes mellitus do tipo 2 é mais frequente aos 40 anos e aumenta a incidência aos 60 anos. No estudo realizado por Eriksson et al. 29 com a população geral da Finlândia, $97 \%$ dos pacientes diabéticos possuíam idade

Modelo Linear Generalizado (MGL) para glicemia capilar casual conforme características estudadas em mulheres da reserva indígena de Dourados, Mato Grosso do Sul, Brasil $(n=385)$.

\begin{tabular}{lcc}
\hline Variáveis & B * (IC95\%) & Valor de $\mathbf{p}$ \\
\hline Intercept & $49,45(28,90-70,00)$ & $<0,001$ \\
Idade (anos) & $0,74(0,37-1,12)$ & $<0,001$ \\
Etnia & 1,00 & - \\
Guarani (referência) & $9,54(1,36-17,73)$ & 0,022 \\
Terena & $0,30(0,11-0,49)$ & 0,002 \\
PAS & &
\end{tabular}

* Os valores de B foram ajustados pelas demais variáveis presentes no modelo.

IC95\%: intervalo de 95\% de confiança; PAS: pressão arterial sistólica. 
superior a 40 anos. A American Diabetes Association (ADA) 22 preconiza a investigação do diabetes mellitus do tipo 2 em indivíduos com idade acima dos 45 anos a cada três anos ou mais precocemente em indivíduos assintomáticos com fatores de risco. As medianas de glicemia capilar casual aumentaram de acordo com a idade nas mulheres indígenas da reserva de Dourados. Fato semelhante foi observado na população indígena nacional em que as médias de glicemia casual aumentaram proporcionalmente à idade 14 .

As medianas de glicemia reduziram com o aumento da escolaridade. Esse resultado foi semelhante a outros estudos 30,31. No inquérito nacional VIGITEL, realizado em 2012 com a população das 26 capitais brasileiras e do Distrito Federal, 12,1\% das pessoas com menos de 8 anos de escolaridade referiram diabetes mellitus contra $3,8 \%$ das pessoas com mais de 12 anos de estudo 30 . No Estado de São Paulo, 6,9\% das mulheres com diabetes mellitus referiram nenhuma escolaridade, e 2,6\%, entre 8 a 10 anos de escolaridade 31 . De acordo com o Censo Escolar, $60,8 \%$ dos indígenas possuem entre 1 e 4 anos de estudo ${ }^{32}$. Possivelmente, isso ocorra devido aos indivíduos de maior escolaridade praticarem mais atividade física e possuírem hábitos alimentares saudáveis 30 .

Apesar de a comparação entre as medianas de glicemia capilar casual não apresentar diferenças estatisticamente significativas segundo o nível socioeconômico, 42,8\% das mulheres que apresentaram glicemia capilar casual alterada encontravam-se no tercil 3, de melhor poder aquisitivo. No estudo realizado na província de Kalutara, no Sri Lanka, em uma população com diferentes níveis socioeconômicos, o maior percentual de diabéticos $(17,4 \%)$ encontrava-se no nível econômico mais baixo ${ }^{33}$. É importante ressaltar que o contato próximo dos indígenas com a população urbana leva a mudanças culturais importantes, incorporando hábitos da população não indígena ${ }^{14}$. Dentre esses hábitos, destacamos a compra de alimentos industrializados que podem exercer influência no perfil glicêmico. $\mathrm{Na}$ reserva indígena de Dourados, a incorporação de novos hábitos é bastante evidente, uma vez que a reserva se localiza na periferia da cidade 16 .

O tabagismo é um importante fator para o desenvolvimento de doenças crônicas como as doenças cardiovasculares 34 . Entre as mulheres entrevistadas, o tabagismo foi referido por $11,9 \%$. Esse percentual foi superior ao investigado nas mulheres das 26 capitais brasileiras e Distrito Federal $(8,6 \%)$ 30. No estudo realizado na aldeia Jaguapiru entre 2007 e 2008, a prevalência de fumantes entre homens e mulheres foi de $19 \%$. Apesar de a comparação das medianas de glicemia capilar casual entre os tabagistas e os não tabagistas não apresentar diferença estatística significativa na população deste estudo, o tabagismo está associado à resistência à insulina, e tabagistas com diabetes mellitus apresentam maior morbidade e mortalidade associadas às complicações vasculares 35 .

$\mathrm{O}$ aumento do índice de massa corporal e a obesidade central estão estreitamente associados à ocorrência de diabetes mellitus 36 . A adiposidade abdominal leva à resistência à insulina por mecanismos ainda não esclarecidos, mas evidências científicas relatam que o excesso de peso sintetiza e ativa proteínas inflamatórias que influenciam na via intracelular da insulina 37 . As médias do IMC e CC foram superiores quando comparadas às das mulheres indígenas Guarani e Tupiniquins do Espírito Santo, Região Sudeste do Brasil, e às dos indígenas Yanomami do Estado de Roraima 24,38. Apesar de os indígenas Tupiniquins sofrerem bastante influência da cultura urbana, essa etnia ainda vive da agricultura familiar tradicional e, raramente, trabalha nas empresas localizadas nas cidades 39 . Já os indígenas Yanomami praticam atividades físicas laborativas necessárias para a sua sobrevivência 19 . Dentre as pessoas com IMC $\geq 30 \mathrm{~kg} / \mathrm{m}^{2}, 5 \%$ apresentaram glicemia capilar casual alterada no presente estudo, percentual superior ao da população indígena nacional $(2,6 \%) 40$. A alimentação rica em carboidratos e gordura pode contribuir para o aumento da obesidade entre os indígenas e, consequentemente, influenciar no perfil glicêmico 41 . Além disso, os indígenas confinados em área restrita tiveram a alteração da economia de subsistência e convivem com o sedentarismo, que é um dos fatores que explicam a obesidade nessa população 14 .

O diabetes mellitus é considerado um fator de risco para doenças cardiovasculares e, frequentemente, está associado à hipertensão arterial 42 . Raça, idade, sexo, proteinúria maciça, aumento do IMC e tempo de evolução do diabetes mellitus são fatores determinantes para o aumento da pressão arterial, principalmente a sistólica 43,44 . No Rio Grande do Sul, um estudo realizado com indivíduos diabéticos demonstrou que a hipertensão arterial está presente em 83,3\% das mulheres 45 . Entre as mulheres da reserva indígena de Dourados, $64,2 \%$ com glicemia capilar casual alterada apresentaram PAS $\geq 140 \mathrm{mmHg}$ e PAD $\geq$ $90 \mathrm{mmHg}$.

Os indivíduos com histórico familiar de primeiro grau para diabetes mellitus possuem um risco elevado de desenvolver essa doença 46. Apesar de não haver, neste estudo, diferença estatística significativa entre a mediana de glicemia capilar casual conforme o histórico familiar 
pesquisas têm demonstrado que, além dos fatores comportamentais relacionados com o ambiente familiar, fatores genéticos têm contribuído para a ocorrência do diabetes mellitus 47 . No presente estudo, $64,2 \%$ das mulheres com glicemia capilar casual alterada não apresentaram histórico familiar para diabetes mellitus. A falta de associação entre o perfil glicêmico e o histórico familiar de diabetes mellitus pode ser explicada pelo fato de a população indígena apresentar um estado de transição epidemiológica no qual o surgimento das DCNT está relacionado com as alterações comportamentais 4 .

$\mathrm{O}$ presente estudo apresentou algumas limitações. Dentre essas, a comunicação devido à língua falada pela população indígena, porém esse fato foi minimizado pela utilização do indígena tradutor sempre que necessário. Outra limitação foi a metodologia utilizada, glicemia capilar casual que sugere a presença de diabetes mellitus. O resultado do nível socioeconômico pode ter sido influenciado pela doação de bens para esse grupo populacional. É importante esclarecer que os resultados aqui apresentados não podem ser inferidos a toda a população indígena dada à diversidade de etnias e características delas.

\section{Colaboradores}

G. A. Freitas elaborou o artigo, e participou da revisão final do artigo para publicação. M. C. C. Souza e R. C. Lima colaboraram na revisão do artigo e aprovação da versão final para publicação.

\section{Conclusão}

A prevalência de glicemia capilar casual alterada sugestiva de diabetes mellitus no presente estudo foi superior a das mulheres indígenas em nível nacional. A idade e a hipertensão arterial estiveram associadas ao perfil glicêmico das mulheres indígenas de Dourados. Outro fator importante é a questão cultural, uma vez que a glicemia capilar casual foi maior na etnia que possui um contato mais intenso com a população não indígena.

Portanto, a população indígena de Dourados, necessita de uma monitorização com vistas a prevenir a ocorrência de distúrbios metabólicos e de complicações relacionadas à hiperglicemia, bem como de modificações comportamentais com o auxílio de intervenções educativas.

\section{Agradecimentos}

Agradecimento aos indígenas da Reserva Indígena do Município de Dourados. 


\section{Referências}

1. Ferreira S. Aspectos epidemiológicos do diabetes mellitus e seu impacto no indivíduo e na sociedade. E-book. Diabetes na prática clínica. http:// www.diabetesebook.org.br/ (acessado em 23/ Out/2013)

2. International Diabetes Federation. Number of people with diabetes. http://www.idf.org/diabetesatlas/data-visualisations (acessado em 07/ Mar/2014).

3. Portal Brasil. Número de pessoas com diabetes aumenta $40 \%$ em seis anos. http://www.brasil.gov. $\mathrm{br} /$ saude/2013/11/numero-de-pessoas-com-diabetes-aumenta-40-em-seis-anos (acessado em 21/ Jun/2015)

4. Coimbra Jr. CEA, Santos RV, Escobar AL. Epidemiologia e saúde dos povos indígenas no Brasil. Editora Fiocruz; 2003.

5. Manitoba Centre for Health Policy. Profile of Métis health status and healthcare utilization in Manitoba: a population-based study. http://www. mmf.mb.ca/docs/metis_health_status_report.pdf (acessado em 15/Jun/2015).

6. Ravussin E, Valencia ME, Esparza J, Bennett PH, Schulz LO. Effects of a traditional lifestyle on obesity in Pima Indians. Diabetes Care 1994; 117: 1067-74.

7. Bennett P, Burch TA, Miller M. Diabetes mellitus in American (Pima) Indians. Lancet 1971; 2:125.

8. Santos JL, Perez Bravo F, Carrasco E, Calvillan M, Albala C. Low prevalence of type 2 diabetes despite a high average body mass index in the Aymara $\mathrm{Na}$ tives from Chile. Nutrition 2001; 17:305-9.

9. Vieira-Filho JPB. Análise das glicemias dos índios das aldeias Suruí, Gaviões e Xikrín. AMB Rev Assoc Med Bras 1975; 21:252-5.

10. Vieira-Filho JPB. O diabetes mellitus e as glicemias de jejum dos índios Caripuna e Palikur. AMB Rev Assoc Med Bras 1977; 123:175-8.

11. Oliveira GF, Oliveira TRR, Rodrigues FF, Corrêa LF, Ikejiri AT, Casulari LA. Prevalência de diabetes melito e tolerância à glicose diminuída nos indígenas da Aldeia Jaguapiru, Brasil. Rev Panam Salud Pública 2011; 29:315-21.

12. Oliveira GF, Oliveira TR, Ikejiri AT, Andraus MP, Galvão TF, Silva MT, et al. Prevalence of hypertension and associated factors in an indigenous community of Central Brazil: a population-based study. PloS One 2014; 9:86278.

13. Dal Fabbro AL, Franco LJ, da Silva AS, Sartorelli DS, Soares LP, Franco L, et al. High prevalence of type 2 diabetes mellitus in Xavante Indians from Mato Grosso, Brazil. Ethn Dis 2014; 24:35-40.

14. Coimbra CE Jr., Santos RV, Welch JR, Cardoso AM, de Souza MC, Garnelo L, et al. The First National Survey of Indigenous People's Health and Nutrition in Brazil: rationale, methodology, and overview of results. BMC Public Health 2013; 13:52.
15. Instituto Brasileiro de Geografia e Estatística. Censo demográfico de 2010: características gerais dos indígenas. Resultados do universo. Rio de Janeiro: Instituto Brasileiro de Geografia e Estatística; 2010.

16. Aylwin J. Os direitos dos povos indígenas no Mato Grosso do Sul, Brasil: confinamento e tutela no século XXI. http://servindi.org/pdf/Iwgia_Informe3. pdf (acessado em 24/Nov/2014).

17. Santana Junior JR. Produção e reprodução indígena: o vir e o porvir na reserva de Dourados/MS Revista de Geografia Agrária 2010; 5:203-36.

18. Fundação Nacional de Saúde. Distrito sanitário do Mato Grosso do Sul, 2010. http://sis.funasa.gov. br/transparencia_publica/siasiweb/Layout/quantitativo_de_pessoas_2010.asp\# (acessado em 05/ Abr/2013).

19. Ministério da Saúde. Sistema de Informações da Atenção à Saúde Indígena. Demografia dos povos indígenas. http://dw.saude.gov.br/gsid (acessado em 20/Mai/2013).

20. Chobanian AV, Bakris GL, Black HR, Cushman WC, Green LA, Izzo JL Jr, et al. The seventh report of the joint national committee on prevention, detection, evaluation, and treatment of high blood pressure: the JNC 7 report. JAMA 2003; 289:2560-71.

21. World Health Organization. Defining the problem of overweight and obesity. In: World Health Organization, editor. Obesity: preventing and managing the global epidemic: report of a Who Consultation. Geneva: World Health Organization; 2000. p. 241-3. WHO Technical Report Series, 894).

22. American Diabetes Association. Standarts of medical care in diabetes. Diabetes Care 2013; 36 Suppl 1:S11-66.

23. Turkman MAA, Silva GL. Modelos lineares generalizados - da teoria à prática. In: VIII Congresso Anual da Sociedade Portuguesa de Estatística. Lisboa: Universidade Técnica de Lisboa; 2000. p. 113-22.

24. Bloch KV, Coutinho ESF, Lobo MSC, Oliveira JEP, Milech A. Pressão arterial, glicemia capilar e medidas antropométricas em uma população yanomámi. Cad Saúde Pública 1993; 9:428-38.

25. Santos KM, Tsutsui MLS, Galvão PPO, Mazzucchetti L, Rodrigues D, Gimeno SGA. Grau de atividade física e síndrome metabólica: um estudo transversal com indígenas Khisêdjê do Parque Indígena do Xingu, Brasil. Cad Saúde Pública 2012; 28:2327-38

26. Henry RE, Burch TA, Bennett PH, Miller M. Diabetes in the Cocopah Indians. Diabetes 1969; 18:33-7. 
27. Stein JH, West KM, Robey JM, Tirador DF, McDonald GW. The high prevalence of abnormal glucose tolerance in Cherokee Indians of North Carolina. Arch Intern Med 1965; 116:842.

28. American Diabetes Association. Economic cost of diabetes in the US in 2007. Diabetes Care 2008; 31:596-615.

29. Eriksson J, Forsén B, Häggblom M, Teppo AM, Groop L. Clinical and metabolic characteristics of type 1 and type 2 diabetes: an epidemiological study from the Närpes community in Western Finland. Diabet Med 1992; 9:654-60.

30. Departamento de Vigilância de Doenças e Agravos não Transmissíveis e Promoção de Saúde, Secretaria de Vigilância em Saúde, Ministério da Saúde. Vigitel Brasil 2012: vigilância de fatores de risco e proteção para doenças crônicas por inquérito telefônico. Brasília: Ministério da Saúde; 2013.

31. Goldenberg P, Franco LJ, Pagliaro H, Rebeca SS, Santos CA. Diabetes mellitus autorreferido no $\mathrm{Mu}$ nicípio de São Paulo: prevalência e desigualdade. Cad Saúde Pública 1996; 12:37-45.

32. Ministério da Educação. Estatísticas sobre a educação escolar indígena no Brasil. http://www.oei. es/quipu/brasil/estadisticas/educ_indigena2007_1. pdf (acessado em 29/Out/2014).

33. Pubudu De Silva A, Padmal De Silva SH, Liyanage IK, Rajapakse LC, Jayasinghe KS, et al. Social, cultural and economic determinants of diabetes mellitus in Kalutara district, Sri Lanka: a cross sectional descriptive study. Int J Equity Health 2012; 11:76

34. World Health Organization. WHO report on the global tobacco epidemic, warning about the dangers of tobacco. Geneva: World Health Organization; 2011.

35. Nunes E. Consumo de tabaco. Efeitos na saúde. Revista Portuguesa de Medicina Geral e Familiar 2006; 22:225-44.

36. Must A, Spadano J, Coakley EH, Field AE, Colditz G, Dietz WH. The disease burden associated with overweight and obesity. JAMA 1999; 282:1523-9.

37. Holland WL, Bikman BT, Wang LP, Yuguang G, Sargent KM, Bulchand S, et al. Lipid-induced insulin resistence mediated by the proinflamatory receptor TLR4 requires saturated fatty acid-induced ceramide biosynthesis in mice. J Clin Invest 2011; 121:1858-70.
38. Oliveira AR, Mourao-Junior CA, Oliveira CM, Krieger JE, Mill JG, Pereira AC. Body mass index, waist circumference, body adiposity index, and risk for type 2 diabetes in two populations in Brazil: general and amerindian. PloS One 2014; 9:100223.

39. Meyerfreund D. Estudo da hipertensão arterial e de outros fatores de risco cardiovascular nas comunidades indígenas do Espírito Santo-BR [Tese de Doutorado]. Vitória: Programa de Pós-graduação em Ciências Fisiológicas, Universidade Federal do Espírito Santo; 2006.

40. Associação Brasileira de Pós-graduação em Saúde Coletiva. I Inquérito Nacional de Saúde e Nutrição dos Povos Indígenas. Relatório final. Rio de Janeiro: Associação Brasileira de Pós-graduação em Saúde Coletiva; 2010

41. Flemig MM, Santos RV, Coimbra Jr. CEA. Blood pressure levels of the Suruí and Zoró Indians of the Brazilian Amazon: group- and sex-specific effects resulting from body composition, health status, and age. Hum Biol 1991; 63:835-61.

42. Gerstein HC, Pogue J, Mann JFE, Lonn E, Dagenais GR, McQueen M, et al. The relationship between dysglycemia and cardiovascular and renal risk in diabetic and non-diabetic participants in the HOPE study: a prospective epidemiological analysis. Diabetologia 2005; 48:1749-55.

43. Kamnnel WB, Schwartz MJ, McNamara PM. Blood pressure and risk of coronary heart disease: the Framingham Study. Dis Chest 1969;56:43-52.

44. Koivisto VA, Yki-Järvinen H, DeFronzo RA. Physical training and insulin sensitivity. Diabetes Metab Rev 1986; 1:445-81.

45. Picon PX, Zanatta CM, Gerchman F, Zelmanovitz T, Gross JL, Canani LH. Análise dos critérios de definição da síndrome metabólica em pacientes com diabetes melito tipo 2. Arq Bras Endocrinol Metab 2006; 550:250-63.

46. Fremmeng Meigs JB, Cupples LA, Wilson PW. Parental transmission of type 2 diabetes: the Framingham Offspring Study. Diabetes 2000; 49: 2201-07.

47. Sladek R, Rocheleau G, Rung J, Dina C, Shen L, Serre D, et al. A genome-wide association study identifies novel risk loci for type 2 diabetes. Nature 2007; 445:881-5. 


\section{Abstract}

This study's objective was to estimate the prevalence of abnormal casual capillary blood glucose, suggestive of diabetes mellitus, and the associations with socioeconomic, demographic, anthropometric, and clinical factors in indigenous women in Dourados, Mato Grosso do Sul State, Brazil. This was a cross-sectional study with a probabilistic sample of women 18 to 59 years of age in the Bororó and Jaguapiru villages. A standardized questionnaire was used to collect data, and statistical analysis used the Kruskal-Wallis, Mann-Whitney, and generalized linear model tests. Of the 385 women, 7\% showed abnormal casual capillary blood glucose, suggestive of diabetes mellitus. In the final generalized linear model, the following remained associated with the outcome: age $(p<0.001)$, systolic blood pressure ( $p=0.002)$, and ethnicity $(p=0.022)$. The study shows the need for behavior changes, using educational interventions as a strategy for the prevention and control of diabetes mellitus.

Hyperglycemia; Diabetes Mellitus; Women's Health; Indigenous Population

\section{Resumen}

El objetivo de este estudio fue estimar la prevalencia de glucemia capilar casual alterada, indicadora de diabetes mellitus, y su asociación con los factores socioeconómicos, demográficos, antropométricos y clínicos en mujeres indígenas del municipio de Dourados, MS. Se trata de un estudio transversal, con una muestra probabilística compuesta por mujeres de 18 a 59 años, residentes en las aldeas Bororó y Jaguapiru. Se utilizó un cuestionario estandarizado para la recogida de información, y el análisis estadístico, realizado mediante los test Kruskal Wallis, Mann Whitney y el modelo lineal generalizado. Entre las 385 mujeres, un 7\% presentaron glucemia capilar casual alterada indicadora de diabetes mellitus. En el modelo lineal generalizado, permanecieron asociadas en el modelo final las variables edad $(p<0,001)$, presión arterial sistólica $(p=0,002)$ y etnia $(p=0,022)$. Este estudio demuestra la necesidad de cambios comportamentales, con ayuda de intervenciones educativas como estrategia de prevención y control de la diabetes mellitus.

Hiperglucemia; Diabetes Mellitus; Salud de la Mujer; Población Indígena
Recebido em 18/Fev/2015

Versão final reapresentada em 26/Ago/2015 Aprovado em 18/Set/2015 